\title{
Vieillissement et rapports entre générations
}

\section{Rémi Lenoir}

\author{
Université de Paris 1, Centre de Sociologie, d'Éducation et de la Culture
}

Résumé : Des travaux récents sur l'évolution des salaires selon l'âge et les catégories socio-professionnelles mettent en cause une représentation des effets du vieillissement que beaucoup de sociologues partagent : assimilant hâtivement la distribution des salaires moyens touchés, à un moment donné, par des individus d'âge différent à l'évolution moyenne de ces rémunérations au cours de la vie des individus, ils observent que les salaires baissent au-delà d'un certain âge et que cette diminution intervient d'autant plus tôt qu'on descend dans la hiérarchie sociale. Au contraire, l'analyse des courbes longitudinales des salaires fait apparaître qu'avec l'âge les salaires de toutes les catégories sociales tendent à augmenter et que, si l'accroissement moyen des salaires individuels baisse, cette diminution est relativement plus forte chez les cadres que chez les ouvriers (Barge et Payen, 1981). Outre qu'elle permet de redresser une erreur incontestable, l'étude longitudinale des salaires permet de décrire avec la précision nécessaire l'évolution de la valeur d'un individu sur le marché du travail, contribution essentielle à une analyse des trajectoires professionnelles. Il reste qu'on ne saurait abandonner pour autant l'examen des courbes transversales qui, à défaut de décrire l'évolution des salaires selon lâge, établissent un état de la répartition des rémunérations entre les générations et les catégories socio-professionnelles. On peut se demander si une étude sur le vieillissement lui-même peut se passer d'une telle information, car ce processus ne saurait être assimilé à l'avance en âge, sous peine de naturaliser une donnée qui n'existe que socialement définie. En effet, la vieillesse n'est pas un attribut inéluctablement lié à l'avance en âge, tel que l'enregistre l'état civil ; elle est l'objet d'une manipulation sociale qui, précisément, met en relation les générations et les classes sociales.

Date de publication : 9/7/2021

Dossier : Sociologie et statistique

Comment citer : $10.52983 /$ crev.vi0.93

Licence: Cambouis publie ses contenus selon les termes de la Licence Creative Commons Attribution - Pas d'Utilisation Commerciale - Pas de Modification 4.0 International. Les auteurices gardent leurs droits de propriété intellectuelle pleine et entière sur leurs articles. 


\title{
Vieillissement et rapports entre générations
}

\section{Rémi Lenoir}

\author{
Université de Paris 1, Centre de Sociologie, d'Éducation et de la Culture
}

Des travaux récents sur l'évolution des salaires selon l'âge et les catégories socio-professionnelles mettent en cause une représentation des effets du vieillissement que beaucoup de sociologues partagent : assimilant hâtivement la distribution des salaires moyens touchés, à un moment donné, par des individus d'âge différent à l'évolution moyenne de ces rémunérations au cours de la vie des individus, ils observent que les salaires baissent au-delà d'un certain âge et que cette diminution intervient d'autant plus tôt qu'on descend dans la hiérarchie sociale. Au contraire, l'analyse des courbes longitudinales des salaires fait apparaître qu'avec l'âge les salaires de toutes les catégories sociales tendent à augmenter et que, si l'accroissement moyen des salaires individuels baisse, cette diminution est relativement plus forte chez les cadres que chez les ouvriers (Barge et Payen, 1981). Outre qu'elle permet de redresser une erreur incontestable, l'étude longitudinale des salaires permet de décrire avec la précision nécessaire l'évolution de la valeur d'un individu sur le marché du travail, contribution essentielle à une analyse des trajectoires professionnelles. Il reste qu'on ne saurait abandonner pour autant l'examen des courbes transversales qui, à défaut de décrire l'évolution des salaires selon l'âge, établissent un état de la répartition des rémunérations entre les générations et les catégories socio-professionnelles. On peut se demander si une étude sur le vieillissement lui-même peut se passer d'une telle information, car ce processus ne saurait être assimilé à l'avance en âge, sous peine de naturaliser une donnée qui n'existe que socialement définie. En effet, la vieillesse n'est pas un attribut inéluctablement lié à l'avance en âge, tel que l'enregistre l'état civil ; elle est l'objet d'une manipulation sociale qui, précisément, met en relation les générations et les classes sociales.

Des travaux récents sur l'évolution des salaires selon l'âge et les catégories socio-professionnelles mettent en cause une représentation des effets du vieillissement que beaucoup de sociologues partagent : assimilant hâtivement la distribution des salaires moyens touchés, à un moment donné, par des individus d'âge différent à l'évolution moyenne de ces rémunérations au cours de la vie des individus, ils observent que les salaires baissent au-delà d'un certain âge et que cette diminution intervient d'autant plus tôt qu'on descend dans la hiérarchie sociale. Au contraire, l'analyse des courbes longitudinales des salaires fait apparaître qu'avec l'âge les salaires de toutes les catégories sociales tendent à augmenter et que, si l'accroissement moyen des salaires individuels baisse, cette diminution est relativement plus forte chez les cadres que chez les ouvriers (Barge et Payen, 1981). Outre qu'elle permet de redresser une erreur incontestable, l'étude longitudinale des salaires permet de décrire avec la précision nécessaire l'évolution de la valeur d'un individu sur le marché du travail, contribution essentielle à une analyse des trajectoires professionnelles. Or nombre de conduites des individus s'expliquent par la pente, la régularité et la durée de telles trajectoires (Bourdieu, 1979, p. 122-126)․․ Aussi des études de ce type devraient-elles être systématiquement entreprises et étendues à 
d'autres secteurs comme cela l'est déjà par exemple pour la scolarisation. Elles pourraient l'être en particulier à l'équipement et au patrimoine des ménages ainsi qu'à la fréquentation des équipements collectifs, autant de moyens et d'instances par lesquels les structures des rapports entre les groupes sociaux tendent à se reproduire.

Il reste qu'on ne saurait abandonner pour autant l'examen des courbes transversales qui, à défaut de décrire l'évolution des salaires selon l'âge, établissent un état de la répartition des rémunérations entre les générations et les catégories socio-professionnelles. Une telle statistique est aussi pertinente pour le sociologue, car elle met en relation les générations entre elles au sein de chaque catégorie sociale sous un rapport déterminé, en l'occurrence le salaire.

On peut se demander si une étude sur le vieillissement lui-même peut se passer d'une telle information, car ce processus ne saurait être assimilé à l'avance en âge, sous peine de naturaliser une donnée qui n'existe que socialement définie. En effet, la vieillesse n'est pas un attribut inéluctablement lié à l'avance en âge, tel que l'enregistre l'état civil ; elle est l'objet d'une manipulation sociale qui, précisément, met en relation les générations et les classes sociales. On le voit par exemple sur le marché du travail. En ce domaine, la hiérarchie des formes de vieillissement paraît reproduire à sa manière la hiérarchie sociale et semble respecter, si l'on peut dire, la « hiérarchie » jusqu'à l'intérieur des entreprises. Selon les employeurs, le « handicap » le plus lourd des travailleurs vieillissants est « l'affaiblissement » des facultés d'adaptation à des tâches, des méthodes ou des techniques nouvelles ; viennent ensuite la « perte de vitesse », la « perte des forces », puis celle de la « vivacité intellectuelle », de « l'habileté », de la « mémoire » et, en dernier lieu, «l'inaptitude au commandement ». En d'autres termes, cela signifie que la diminution avec l'âge des qualités estimées nécessaires par les employeurs pour l'exercice des diverses activités professionnelles ou, si l'on veut, l'âge à partir duquel les différentes catégories sociales commencent à « vieillir » sur le marché du travail est plus précoce pour les membres des classes les plus basses : pour les chefs d'entreprise, les manœuvres sont considérés comme productifs à $100 \%$ jusqu'à lâge moyen de 51,4 ans seulement, les ouvriers qualifiés jusqu'à 55 ans, les agents de maîtrise jusqu'à 55,9 ans, les contremaîtres d'atelier et les cadres jusqu'à 57,9 ans, les comptables jusqu'à 60,3 ans ${ }^{2}$.

On saisit à travers cette évaluation différentielle de la « productivité » des diverses catégories de travailleurs effectuée par les chefs d'entreprises, c'est-à-dire par des agents socialement intéressés à imposer une définition du vieillissement, id est une valeur sur le marché du travail, que le vieillissement est mesuré, sur ce marché particulier, moins à « l'échelle des âges » qu’à celle de critères dont l'imposition est fonction des rapports de force entre les différentes catégories de vendeurs et d'acheteurs de la force de travail. La force de cette imposition, on peut en avoir une idée approximative lorsqu'on sait que les employés s'appliquent à eux-mêmes les catégories de jugement que les employeurs utilisent pour évaluer le vieillissement de leurs salariés ; alors que $73 \%$ des ouvriers estiment que «l'âge limite de leur pleine capacité professionnelle » est 60 ans, cette proportion passe à $66 \%$ pour les employés et les cadres moyens et n'est que de $45 \%$ pour les cadres supérieurs et les professions libérales (Monnier, 1979, p. 821).

\section{La notion de vieillissement différentiel}

C'est un lieu commun que de reconnaître que les individus « vieillissent » plus ou moins rapidement ( «on a lâge de ses artères »). La notion de vieillissement social différentiel trouve son fondement dans les résultats des études qui mesurent les « inégalités sociales » devant l'usure physique et la longueur de
2 Enquête menée en 1961 par l'IFOP auprès de 200 chefs d'entreprises et directeurs de personnel de grandes et moyennes entreprises privées (IFOP, 1962, p. 99). Sur la relation entre l'âge et la productivité, voir Chesnais (1978). Pour les cadres eux-mêmes, si leurs compétences «techniques » tendent à se dévaluer avec l'âge, leur compétence « directoriale »aptitude à faire travailler les autres - peut s'accroître au cours de leur vie professionnelle (Perrucci et Rothman, 1969). 
la vie biologique (Calot et Febvay, 1965 ; Desplanques, 1976) mais aussi plus généralement devant la durée de la vie sociale comme en témoignent, entre autres indicateurs, l'écart des âges de mise à la retraite de l'activité professionnelle ou la différence des âges d'entrée dans les maisons de retraite selon les classes sociales (Gaullier, 1979 ; Mizrahi, Mizrahi et Moyse, 1977).

Une telle notion permet de rompre avec l'abstraction statistique des tranches d'âge dans la mesure où sont prises en compte les classes sociales que les démographes et même les sociologues tendent à évacuer le plus souvent au profit de classes d'âge socialement indéterminées, comme les «vieux », les « personnes âgées de soixante-cinq ans et plus », etc. ${ }^{3}$ En effet, simple « continuum de relativité », selon l'expression de B. L. Whorf, l'âge n'est pas a priori divisé en catégories et, le serait-il, que la question de la correspondance entre les « divisions arithmétiques » que l'âge abstrait donné par l'état civil rend possible et les « groupes sociaux désignés en termes d'âge » ne s'en poserait pas moins (Halbwachs, 1972, p. 330-334).

La notion d'âge elle-même, celle qu'on exprime en nombre d'années, est le produit d'une pratique sociale déterminée : mesure abstraite dont le degré de précision avec lequel elle s'impose s'explique surtout par des nécessités de la pratique administrative (l'identification des individus, le nom et le lieu d'habitation ne suffisant plus), lâge de l'état civil comme critère de classement est apparu en France au seizième siècle, lors de la généralisation de l'inscription de la naissance sur les registres paroissiaux (Ariès, 1958, p. 2) ${ }^{4}$. Sans doute le recours à l'âge comme catégorie de classement s'explique-t-il par le fait qu'il constitue la « variable rêvée du statisticien » selon l'expression de Laurent Thévenot : numérique, quantitative, continue, universelle, «bref naturellement mathématique ». La variable âge se mesure au nombre des années (Thévenot, 1979, p. 14).

Or, on le sait pourtant, l'effet du temps varie selon les classes sociales comme l'attestent par exemple la durée et la courbe des carrières dans les différentes professions : au cursus professionnel instable des manœuvres (ils font « trente-six métiers ») qui tend à se détériorer rapidement avec l'usure physique et à la carrière « sans avenir » des ouvriers qui sont déjà à trente ans ce qu'ils seront à soixante ${ }^{5}$, s'opposent la stabilité de l'emploi et les petites promotions à « l'ancienneté » des petits fonctionnaires, la «carrière » proprement dite des cadres moyens avec ses promotions au choix et surtout celle des cadres supérieurs, constamment ascendante sous le rapport des « responsabilités ».

Mais si la notion de vieillissement social différentiel est l'instrument qui permet de rompre avec les abstractions que constituent les classes d'âge socialement indifférenciées, il reste que la logique de son usage ne s'éloigne guère du sens commun et ne fait que désigner ce qu'il s'agit d'expliquer. Sans doute permet-elle de réintroduire les classes sociales dans l'analyse, mais son emploi n'aboutit le plus souvent qu'à classer des pratiques selon l'âge et la classe sociale : en croisant par exemple les deux variables « personnes âgées » et classes sociales, le sociologue construit des classes sociales de « personnes âgées » et continue ainsi à faire de l'âge chronologique une propriété substantielle, indépendante de l'ensemble des propriétés dans lequel elle prend sens. Il ne suffit pas, en effet, d'admettre l'existence de cycles de vie propres à chaque classe sociale pour échapper à une conception naturaliste du vieillissement selon laquelle la vieillesse survient, mais sous des formes et à des moments différents selon les classes, lorsque, notamment, les forces physiques déclinent et «obligent » à la retraite. Une telle représentation du vieillissement social reste encore trop proche du modèle qui la fonde, à savoir le vieillissement physique qui, comme l'observe Maurice Halbwachs, se produit « tout au cours de la vie, par transitions insensibles » (Halbwachs,
3 On peut voir un exemple idéal-typique de la démarche du statisticien dans la présentation qu'Alfred Sauvy fait de la notion de vieillissement démographique : « On se trouve, écrit Alfred Sauvy, pour toute population, à un moment donné, devant la répartition :

$\mathrm{P}=\mathrm{J}+\mathrm{A}+\mathrm{V}$, où $\mathrm{P}$ représente la population totale, $\mathrm{J}$ le nombre de jeunes, A le nombre d'adultes, V le nombre de vieux. Ces trois groupes permettent de calculer trois indices de vieillissement :

- le rapport V/P du nombre des vieux à la population totale,

- le rapport V/J du nombre des vieux au nombre des jeunes,

- le rapport V/A du nombre des vieux au nombre des adultes » (Sauvy, 1963, p. 371).

4 On peut rappeler que les catégories d'âge ne sont pas neutres, comme en témoignent les catégorisations des premiers recensements de population. Ainsi celui de Trévise, effectué en 1384, distingue deux catégories : les hommes de plus et de moins de quatorze ans, « les religieux et les domestiques étant comptés à part » pour la raison que ces derniers, comme les enfants de moins de quatorze ans et les femmes, quel que soit leur âge (pendant longtemps exclues de tout recensement), ne payant pas d'impôts et ne portant pas les armes, n'étalent des « biens à recenser » (Mols, 1954, p. 170-171).

5 Ainsi, par exemple, l'âge limite d'embauche est fréquemment de trente ans pour les postes d'ouvriers spécialisés de l'industrie électronique et du textile (Laville et al, 1973, p. 3). 
1972, p. 270 $)^{6}$. Dans cette logique, le vieillissement social reste défini comme un processus individuel dont la « vitesse » varie selon la valeur des différentes formes de capital détenu par l'individu.

\section{Le vieillissement, un enjeu de luttes}

Une telle conception du vieillissement social tend à occulter le fait que la définition même du vieillissement est l'objet d'une lutte. La vieillesse n'est pas une sorte de propriété substantielle qui advient avec lâge : elle est une catégorie dont la délimitation résulte de l'état (variable) des rapports de force entre les classes et, dans chaque classe, des rapports entre les générations, c'est-à-dire de la distribution du pouvoir et des privilèges entre les classes et entre les générations.

Chacun sait en effet que les limites syncrétiquement perçues par les individus qui séparent l'« âge adulte » de la « jeunesse » ou de la « vieillesse » varient selon les sociétés et selon les groupes à l'intérieur d'une même société. Dans son étude sur « la nuptialité en France pendant et depuis la guerre », Maurice Halbwachs montre, par exemple, comment la définition sociale des âges est fonction de la composition numérique des générations ; la diminution extrêmement sensible, consécutive à la guerre, de la population masculine ayant entre vingt-trois et trente-huit ans, a eu pour effet de « relever les jeunes dans les échelles des âges » dans la mesure où, conduits à occuper des positions laissées vacantes par leurs aînés, ils ont été amenés à exercer des responsabilités qui étaient jusqu'alors « comme au-dessus de leur âge », cette transformation s'est accompagnée d'une redéfinition de l'âge légitime au mariage et plus généralement de lâge auquel les « jeunes » accèdent.

Cette analyse n'accorde-t-elle pas cependant trop d'importance aux phénomènes purement morphologiques, les transformations démographiques exerçant leurs effets différentiellement selon les groupes? Ce qui est en jeu dans la manipulation du classement des âges est la définition des pouvoirs associés aux différents moments du cycle de vie, l'étendue et le fondement du pouvoir variant selon la nature des enjeux, propres à chaque classe ou à chaque fraction de classe, de la lutte entre les générations ${ }^{7}$. On le voit notamment lorsque l'état des rapports de force se modifie, comme le montre l'exemple idéal-typique de l'évolution, dans la seconde moitié du dix-neuvième siècle, des relations entre générations chez les artisans verriers, consécutive à la transformation des techniques de production dans ce secteur (Scott, 1974, p. 100-107). En effet, la mécanisation et la simplification des tâches qui lui est associée ont sapé un des fondements du pouvoir des maîtres verriers, à savoir le monopole de la détention des techniques de fabrication et corrélativement celui de leur transmission : les souffleurs contrôlaient jusqu'alors à leur profit les rapports entre les générations en imposant des temps de formation et des niveaux de compétence à leurs successeurs, « gamins » et « grands garçons », ces appellations désignant autant une position dans l'échelle des âges et dans celle de la profession que, plus généralement, une position sociale (faible rémunération, célibat, etc.). Devant la concurrence des jeunes apprentis, les verriers, après avoir tenté sans succès de retarder l'âge d'accès à l'apprentissage et d'allonger la durée de la formation, ont été contraints à adopter des stratégies défensives en reconnaissant notamment à leurs concurrents les plus dangereux, dont la qualification était égale à la leur, un statut spécial, afin d'en rendre la fonction permanente et du même coup d'empêcher les « garçons », devenus trop « grands », de les évincer.

Ainsi il est exclu de fixer pour les membres d'une même classe sociale, a fortiori pour les individus quelconques, lâge à partir duquel ils deviennent « vieux », c'est-à-dire « trop vieux » pour exercer une activité donnée ou pour
6 À l'inverse, la maladie du siècle dont souffrait la jeunesse bourgeoise de 1830 tenait pour une grande part au fait que les carrières dans les professions libérales ou dans la haute administration étaient bloquées par un recrutement d'hommes relativement jeunes sous Louis XVIII. Lâge d'accès à ces positions (et à ce qui lui est lié, notamment le mariage) était du même coup retardé et les « jeunes » condamnés à une adolescence prolongée (« la vie de bohème ») et, pour reprendre une autre expression de Musset dans $L a$ Confession d'un enfant du siècle, à une « affreuse désespérance » (de Bertier de Sauvigny, 1955, p. 320-323).

7 Georges Duby a montré que la constitution, dans la société aristocratique française du douzième siècle, d'une nouvelle étape bien déterminée de l'existence désignée comme « jeunesse », moment compris entre l'adoubement qui marquait la sortie de l'enfance et le mariage qui définissait l'adulte accompli, était le produit des stratégies familiales de conservation du pouvoir et de préservation du patrimoine des lignées.

L'appartenance à la « jeunesse » était moins fonction de l'âge biologique que de l'âge, fort variable, auquel les héritiers accédaient à la gestion du patrimoine, c'est-à-dire en général à la mort de leur père. En prolongeant la « jeunesse » de leurs enfants, c'est-à-dire en les éloignant du fief (croisade, tournois, etc.), les parents reculaient d'autant l'âge auquel ils étaient considérés comme « vieux ». Les « jeunes » étaient ainsi des chevaliers célibataires voués à l'errance et à l'aventure en attendant le moment où ils pouvaient succéder à leurs parents et se marier (Duby, 1964). 
accéder légitimement à certaines catégories de biens ou de positions. C'est précisément la détermination de cet âge, moment capital où les générations les plus jeunes parviennent à contraindre les générations plus âgées à se retirer des positions de pouvoir, afin de les occuper à leur tour, qui constitue l'enjeu de la lutte entre les générations. Aussi peut-on se demander si la sociologie de la vieillesse, qui prend comme objet une population définie pour l'essentiel par l'âge légal, ou par l'état de vieillissement biologique, ne l'anéantit pas par avance dans la mesure où elle considère comme résolu ce qu'il s'agit justement d'expliquer'.

Mais du fait « qu'on ne sait pas à quel âge, à quel moment de la vie commence la vieillesse », faut-il, à la manière de ces sociologues qui, comme l'observe Pareto, «ne pouvant pas tirer une ligne pour séparer d'une façon absolue les riches et les pauvres », concluaient à l'absence des antagonismes de classes, en déduire qu'il n'existe pas de vieillards (Pareto, 1964, p. 385) ? L'objet de la sociologie de la vieillesse, par exemple, ne consiste pas à définir qui est vieux et qui ne l'est pas ou à dire à partir de quel âge les agents des différentes classes sociales le deviennent, mais à décrire le processus à travers lequel les individus sont socialement désignés comme tels ${ }^{9}$.

La sociologie de la vieillesse représente à cet égard un cas limite dans la mesure où la sociologie de l'objet préconstruit constitue presque exclusivement l'objet même de la recherche, la définition de la vieillesse étant un enjeu de lutte entre les générations mais aussi entre les agents et les institutions en concurrence pour le contrôle de la prise en charge matérielle et culturelle des «personnes âgées $»^{10}$. Le découpage des âges et les définitions des pratiques légitimes qui leur sont associées tiennent en effet à l'apparition d'institutions et d'agents spécialisés, comme on l'a établi, par exemple, à propos de la distinction des premiers âges de la vie, liée au développement du système scolaire. L'invention de « l'enfance », de « l'adolescence » et plus récemment de la « prime enfance » résultent pour une bonne part de l'allongement de la durée des études et de la diffusion de l'école maternelle (Chamboredon et Prévôt, 1973). De même, aujourd'hui, l'invention du «troisième âge », cette nouvelle étape du cycle de vie qui tend à s'intercaler entre la retraite et la vieillesse, est pour l'essentiel le produit de la généralisation des systèmes de retraite et de l'intervention corrélative d'institutions et d'agents qui, en se spécialisant dans le traitement de la vieillesse, contribuent au processus d'autonomisation de la catégorie et du même coup de la population qu'elle désigne ${ }^{11}$.

Un des moyens de rompre avec l'usage substantialiste de l'âge qui revient à faire de ce dernier une entité dotée d'une force propre, indépendante de l'ensemble des propriétés dans lequel il agit, est d'observer la distribution des propriétés qui sont attachées à un moment donné aux différentes catégories d'âge selon les catégories sociales. En effet, les distributions instantanées livrent, de façon très grossière, ce qu'on pourrait appeler l'état des rapports de force entre les générations et les catégories sociales. Ainsi, par exemple, sur le marché du travail, on remarque que plus on monte dans la hiérarchie professionnelle et plus est grand l'écart des salaires entre les moins et les plus âgés, au profit de ces derniers. En outre, l'inégalité des rémunérations entre les catégories sociales varie selon les générations : elle est d'autant plus forte que les générations de salariés sont plus âgées.

Ainsi, en 1975, les rémunérations des salariés âgés de 61 à 65 ans sont 2,1 fois plus élevées que les traitements de ceux qui sont âgés de 26 à 30 ans pour les cadres supérieurs, ce coefficient passant à 1,3 pour les cadres moyens et devenant négatif pour les autres catégories socio-professionnelles : 0,99 pour les employés, 0,96 pour les ouvriers qualifiés et 0,95 pour les manœuvres. La même année les cadres supérieurs de 26 à 30 ans gagnent 2,9 fois plus que les manœuvres du «même âge » et ceux qui ont entre 61 et 65 ans, 6,5 fois plus (Insee, 1980, p. 85) $)^{12}$.
$8 \quad$ Il n'est pas jusqu'aux recueils de données statistiques consacrées aux « personnes âgées » que le chercheur est amené à consulter qui ne participent également à la constitution d'une définition de la vieillesse comme une catégorie en soi et analysable en tant que telle (Banderier, Perrot et Volkoff, 1976 ; Centre d'étude et de recherche sur les coûts, 1980).

9 Cela ne signifie pas que l'âge de l'état civil, « quantité mesurable légalement », qui, selon l'expression de Philippe Ariès (1958), ressort du monde « de l'exactitude et du chiffre », n'ait aucune réalité sociale. Il se rappelle continuellement aux individus (anniversaires, démarches administratives, etc.) et constitue une sorte d'étalon abstrait et omnibus d'identification ou si l'on veut un référent permettant les comparaisons. En outre, la fixation d'un âge légal, par exemple, celui de la majorité à dix-huit ans ou celui de la retraite à soixante-cinq ou soixante ans, n'est pas sans effet sur la lutte entre les générations. Il tend à constituer une sorte de norme officielle avec laquelle les agents doivent compter ( « il faut faire place aux jeunes », etc.).

10 Il en est de même de la délinquance juvénile (Chamboredon, 1971) ou des accidents du travail (Lenoir, 1980).

11 Sur la constitution du champ des agents de la vieillesse, voir Lenoir (1979). On voit aujourd'hui un exemple de cette lutte dans la constitution récente de l'opposition entre le « troisième âge » et le « quatrième âge ». Cette distinction correspond en effet à l'arrivée de nouveaux spécialistes dans le système des agents gérant la vieillesse : en différenciant le « quatrième âge », objet de garderie et de soins physiologiques, et le « troisième âge » comme âge réclamant surtout des soins culturels et psychologiques, ils tentent d'imposer de nouveaux besoins et du même coup le besoin de leurs services.

12 On notera que ces résultats ne sont pas en contradiction avec ceux de l'enquête longitudinale portant sur l'évolution des salaires entre 1970 et 1975 , puisque les écarts de salaires entre générations selon les catégories sociales tendent à se réduire : pour ne donner qu'un exemple, en 1972 les cadres supérieurs de 61 à 65 ans gagnaient en moyenne 7,3 fois plus que les manœuvres de la même catégorie d'âge contre 6,5 en 1975 (Chabanas et Volkoff, 1975, p. 66). 
Quand on sait que la valeur des individus - et en particulier celle des hommes - sur le marché du travail est sans doute l'une des variables essentielles qui agit sur le vieillissement social en raison du poids fonctionnel important que possède l'activité professionnelle dans la définition de la valeur sociale des individus, le vieux manœuvre est, si l'on peut dire, doublement « vieilli », pour ne pas dire dominé : il l'est par rapport au jeune ouvrier (qui peut être son propre fils) dont le salaire est plus élevé et qui est au début d'une trajectoire professionnelle (avec des possibilités de promotion) ; il l'est aussi par rapport aux autres personnes du «même âge » puisqu'il occupe la position (mesurée pour la circonstance par le montant du salaire) relativement la plus basse de toutes.

Il reste que tous les espaces d'activité ne sont pas totalement congruents et il serait très pertinent en conséquence de compléter cette description des rapports objectifs entre les générations grâce aux courbes transversales qui préciseraient en particulier la répartition des biens durables et du patrimoine ainsi que la fréquentation des équipements collectifs selon les générations et les catégories sociales. On se donnerait ainsi le moyen de comprendre notamment des phénomènes diversement baptisés «nouvelle vague », « troisième âge », « crise de la quarantaine », etc. qui désignent en fait, mais de façon euphémique, certains effets de luttes que se livrent les générations et les classes sociales pour l'appropriation des biens et l'occupation des positions que définit tout système social. 


\section{Bibliographie}

ARIÈs Philippe, 1958, L'enfant et la vie familiale sous l'Ancien Régime, Paris, Plon.

Banderier Gaston, Perrot Marguerite et Volkoff Serge, 1976, « Les revenus des personnes âgées », Les collections de l'INSEE, $\mathrm{n}^{\circ} 52$.

BARge Michel et PAYEN Jean-François, 1981, «Vieillissement et salaire : une optique individuelle », Économie et statistique, $\mathrm{n}^{\circ} 139$, p. 3-16.

Bourdieu Pierre, 1979, La distinction, Paris, Éditions de Minuit, 1979.

CALOT Gérard et FeвWAY Maurice, 1965, « La mortalité différentielle suivant le milieu social, présentation d'une méthode expérimentée en France sur la période 1955-1960 », Économie et statistique, vol. 20, nº 11, p. 75159.

CENTRE D'ÉTUdE ET DE RECHERCHE SUR LES COÛTS, 1980, Les revenus des personnes âgées, Paris, CERE.

Chabanas Nicole et Volkoff Serge, 1975, « Les salaires dans l'industrie, le commerce et les services en $1972 »$, Les collections de l'INSEE, $\mathrm{n}^{\circ} 45$.

Chamboredon Jean-Claude et Prévôt Yves, 1973, « Le métier d'enfant. Définition sociale et fonction différentielles de l'école maternelle », Revue française de sociologie, vol. 14, $\mathrm{n}^{\circ}$ 3, p. 295-335.

Снамвoredon Jean-Claude, 1971, « La délinquance juvénile, essai de construction d'objet », Revue française de sociologie, vol. 12, $\mathrm{n}^{\circ} 3$, p. 335-377.

Chesnais Jean-Claude, 1978, «Age, productivité et salaires », Population, $\mathrm{n}^{\circ} 6$.

De Bertier de Sauvigny Guillaume, 1955, La Restauration, Paris, Flammarion.

DesPlanQues Gérard, 1976, « La mortalité des adultes suivant le milieu social 1955-1971 », Les Collections de l'INSEE, $\mathrm{n}^{\circ} 44$.

Duby Georges, 1964, « Les "jeunes" dans la société aristocratique dans la France du Nord-Ouest au XIIe siècle », Annales, vol. 19, n 5, p. 835846.

GaUllier Xavier, 1979, Politique de l'emploi, modes de vie et vieillissement, Paris, Fondation des villes.

IFOP, 1962, « Les travailleurs âgés dans l'entreprise », in Haut comité consultatif de la population et de la famille, Les personnes âgées et l'opinion en France, Paris, La Documentation Française.

INSEE, 1980, « Les salaires dans l'industrie, le commerce et les services en $1975 »$, Les collections de l'INSEE, $\mathrm{n}^{\circ} 82$.

Laville Antoine, Teiger Catherine et Duraffourg Jacques, 1973, Charge de travail et vieillissement, Paris, CNAM.

LENOIR Rémi, 1979, «L'invention du troisième âge », Actes de la recherche en sciences sociales, $\mathrm{n}^{\circ} 26-27$, p. 57-82.

LENOIR Rémi, 1980, « La notion d'accident du travail : un enjeu de luttes », Actes de la recherche en sciences sociales, $\mathrm{n}^{\circ}$ 32-33, p. 77-88.

HalbWachs Maurice, 1972, Classes sociales et morphologie, Paris, Éditions de Minuit.

Mizrahi Andrée, Mizrahi Arié et Moyse Dominique, 1977, Les personnes âgées vivent en institutions, Paris, CREDOC.

Mols Roger, 1954, Introduction à la démographie des villes d'Europe du XlVe et XVIIIe siècle, Duculot, Gembloux / Presses Universitaires de Louvain, Tome 1.

Monnier Alain, 1979, « Les limites de la vie active et la retraite », Population, $\mathrm{n}^{\circ} 4$.

PAREto Vilfredo, 1964, Cours d'économie politique, Genève, Droz, Tome XI. 
Perrucci Robert et Rothman Robert A., 1969, « Obsolescence of knowledge and the professionnal career », dans PERRUCCI Robert et GERSTL Joël E. (eds), The engineers and the social system, New-York, John Wiley, 1969, p. 247-275.

Rosenberg Georges, 1970, The Worker Grows Old, San Francisco, Jossey-Bass Inc. Publishers.

SAUvY Alfred, 1963, « Le vieillissement démographique », Revue internationale des sciences sociales, vol. $\mathrm{XV}, \mathrm{n}^{\circ} 3, \mathrm{p} .371$.

Scотт Joan Wallach, 1974, The Glassworkers of Carmaux, Cambridge, Mass., Harvard University Press.

ThÉvenot Laurent, 1979, « Une jeunesse difficile. Les fonctions sociales du flou et de la rigueur dans les classements », Actes de la recherche en sciences sociales, $\mathrm{n}^{\circ} 26-27, \mathrm{p} .14$. 